\title{
Direct Observation of Nano-porous Materials Using Low Voltage High Resolution SEM
}

Shunsuke Asahina ${ }^{1}$, Yusuke Sakuda ${ }^{1}$ and Osamu Terasaki ${ }^{2}$

1. JEOL Ltd., 3-1-2 Musashino, Akishima, Tokyo, 196-8558, Japan

2. Dept of Materials \& Environmental Chemistry, EXSELENT, Stockholm Univ, Stockholm, Sweden \& Graduate School of EEWS, KAIST, Daejeon, Republic of Korea

Understanding surface fine structures of nano-porous materials and their compositions is essential for controlling the growth of these materials and for the future material design and the utilization of their functions [1]. Technical developments in low acceleration voltage high resolution scanning electron microscopy (LVHR-SEM) have made it possible to acquire invaluable information about them even though the materials are electrically insulating and electron beam sensitive. In this report, we focused on observations with electron beam at ultra-low impact energy in order to acquire information from top surface of nano porous materials.

We have developed a new objective lens called the super hybrid lens (SHL): a compound lens consisting of both magnetic and electrostatic lenses [2]. The SHL is capable of producing a small probe size even at low accelerating voltages (for example $0.7 \mathrm{~nm}$ at $1 \mathrm{keV}$ ); moreover, using beam deceleration mode allows imaging down to $10 \mathrm{eV}$ with high spatial resolution [3]. Both SHL and beam deceleration mode are installed in a JEOL Field Emission SEM (FE-SEM) JSM-7800F Prime.

The sample preparation is also important for LVHR-SEM observation. Especially, we have to avoid contamination, since sample contamination has a negative effect on imaging quality. It is particularly detrimental in low voltage imaging where only the top few nanometers of the structure are probed with the electron beam. The main sources of contamination are from the specimen itself (physically adsorbed gaseous species), the specimen holder and the microscope chamber. Therefore, we selected a highdensity conductive carbon stub for LVHR-SEM observations. This stub was first polished with sandpaper (\#1200) and then with a filter paper to make the surface smooth. It was then cleaned by ultrasonication in alcohol and heated in vacuum. The samples described in this paper were prepared by one of two methods. With the first method, nano particles were dispersed in solution and then a drop was placed on the carbon stub. The stub was then heated in a vacuum oven for $10 \mathrm{~min}$ at $200^{\circ} \mathrm{C}$ and subsequently cooled to room temperature. The second preparation method was employed for dried particles, which were picked with a cotton ball and scattered on a carbon stub that was polished with filter paper and dried on a hot plate at $250^{\circ} \mathrm{C}$.

Mesoporous zeolite LTA [4] images using low landing energy are show at Fig.1. Fig.1 (a) shows low magnification of mesoporous zeolite LTA at $80 \mathrm{eV}$ as landing energy; (b) and (c) show high magnification of mesoporous zeolite LTA at different landing energies such as $80 \mathrm{eV}$ and $500 \mathrm{eV}$. All images clearly show top surface topographical information of mesoporous zeolite LTA. Especially, the image of $80 \mathrm{eV}$ shows less edge effect due to small interaction volume. That is a great feature of LVHRSEM, since it can show fine edges and give high accuracy measurement of nano-porous materials. The images also demonstrate that higher landing voltage results in specimen damage due to electron beam irradiation (see Fig. 1 (c)), whereas lower landing voltage allows observation without any specimen damage (Fig.1 (b)). 
We will also demonstrate the ability of LVHR-SEM to not only obtain high spatial resolution image with minimal electron beam damage, but also to acquire high spatial resolution chemical information by using Energy Dispersive X-ray Spectrometer (EDS) or Soft X-ray Emission Spectrometer (SXES) [6].

\section{References:}

[1] S. Asahina, et al., ChemCatChem. 1038-1048, June 2011.

[2] J. Frosien, et al., J. Vac. Sci. Technol. B7 (6). 1874, 1989.

[3] S. Asahina, et al., Microscopy and Analysis.S12-S14, November 2012.

[4] K. Cho, et al., Solid State Sciences. Vol. 13. (4). 750-756, April 2011

[5] M. Suga et al., Prog. Solid State Chem. 42, 1-21 (2014).

[6] S.Asahina, et al., APL mat. 2, 113317 (2014).

Acknowledgement:

Mesoporous zeolite LTA crystals were kindly provided by Prof Ryong Ryoo (KAIST \& IBS, Korea).
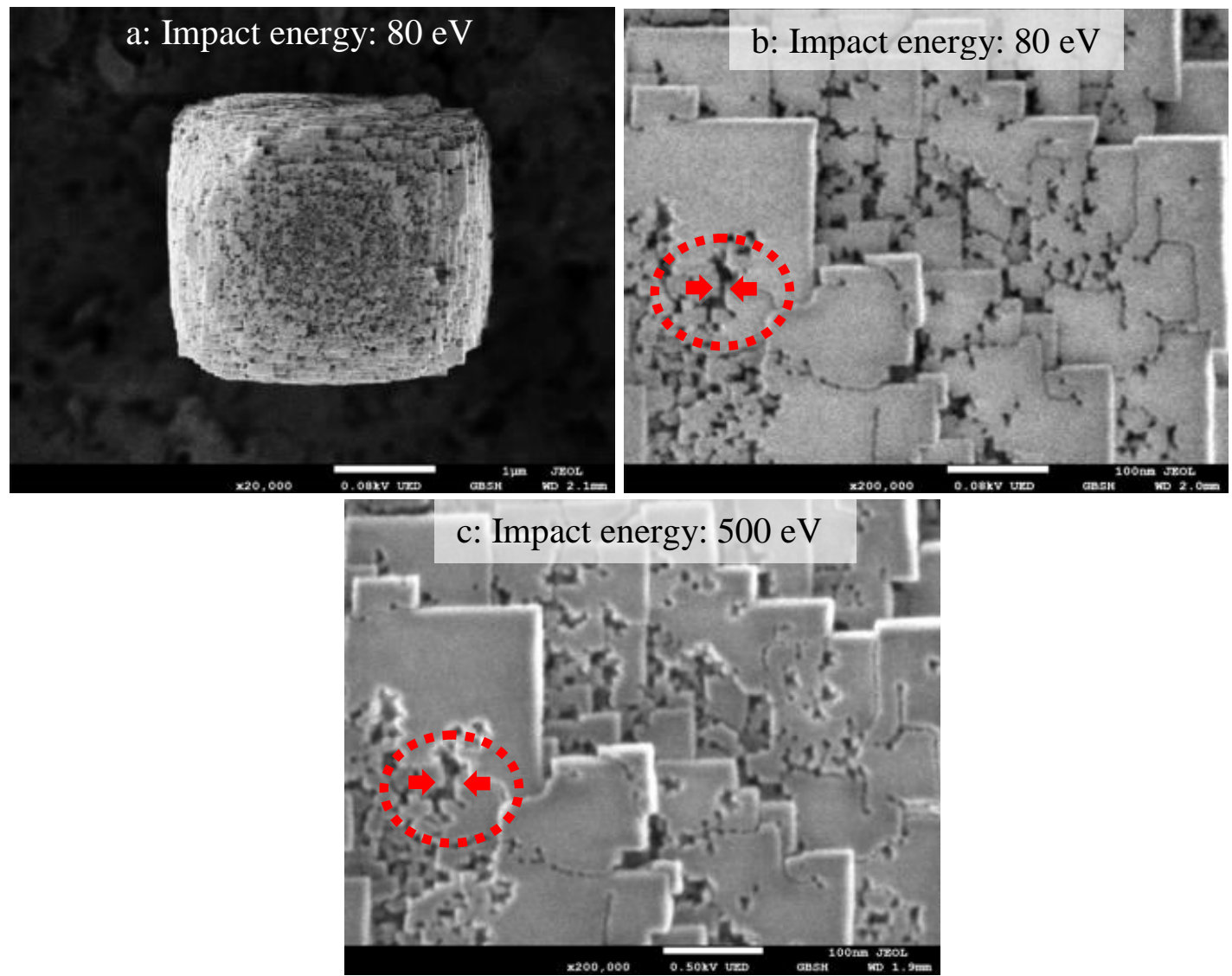

Fig. 1. Low landing energy images at mesoporous zeolite LTA 